\title{
Differential expression of KIT/PDGFRA mutant isoforms in epithelioid and mixed variants of gastrointestinal stromal tumors depends predominantly on the tumor site
}

\author{
Bartosz Wasag ${ }^{1,5}$, Maria Debiec-Rychter ${ }^{1}$, Patrick Pauwels ${ }^{2}$, Michel Stul ${ }^{1}$, Hilde Vranckx ${ }^{1}$, \\ Allan Van Oosterom ${ }^{3}$, Anne Hagemeijer ${ }^{1}$ and Raf Sciot ${ }^{4}$ \\ ${ }^{1}$ Laboratory for Cytogenetics and Molecular Genetics of Human Malignancies, Department for Human \\ Genetics, Catholic University of Leuven, Belgium; ${ }^{2}$ Department of Pathology, University Hospital, Maastricht, \\ The Netherlands; ${ }^{3}$ Department of Oncology, University Hospital Gasthuisberg, Leuven, Belgium; ${ }^{4}$ Laboratory \\ for Morphology and Molecular Pathology, Department of Pathology, Catholic University of Leuven, Leuven, \\ Belgium and ${ }^{5}$ Department of Biology and Genetics, Medical University of Gdansk, Gdansk, Poland
}

\begin{abstract}
Gastrointestinal stromal tumors (GISTs) form a distinctive group of mesenchymal neoplasms, showing differentiation towards the interstitial cells of Cajal. Morphologically, GISTs vary from cellular spindle cell tumors to epithelioid or mixed, epithelioid and spindle cell variants. The genotypic features underlying the morphologic differences of GISTs with vs without epithelioid components are not well defined. Acquisition of activating mutations in KIT and PDGFRA has been reported as alternative oncogenic events in the pathogenesis of GISTs. In this study, a comprehensive KIT and PDGFRA mutational analysis was performed in a group of 28 epithelioid/mixed type tumors, in order to explore whether a specific KIT/PDGFRA mutational status segregates these neoplasms from spindle cell variant GISTs. All GISTs were primary neoplasms, $16(57.1 \%)$ originated from the stomach and $12(42.8 \%)$ from other locations. Histomorphologically, 14 GISTs showed an epithelioid and 14 a mixed cell type pattern. Mutational analysis included KIT exons 9,11,13, and 17, and PDGFRA exons 12 and 18 prescreening by denaturing high-performance liquid chromatography, followed by direct sequencing. Activating mutations of KIT were found in $14(50 \%)$ GISTs, the majority being within exon $11(n=11 ; 39.2 \%)$, and the other comprised exon 9 AY 502-503 duplications $(n=2 ; 7.2 \%)$ and exon 17 Lys $\rightarrow$ Aln $^{822}$ missense mutations $(n=1 ; 3.6 \%)$. Most of the KIT mutant tumors $(n=11 ; 78.6 \%)$ originated from nongastric sites. Seven $(25.0 \%)$ GISTs with no detectable KIT mutations demonstrated PDGFRA mutant isoforms, carrying either D842 V mutations $(n=5)$ or exon 18 deletions $(n=2)$. All GISTs harboring PDGFRA mutant isoforms originated from the stomach. In seven tumors, no detectable mutations were found; all but one of nonmutant tumors initiated from the stomach and exhibited an epithelioid morphology. These findings indicate that the mutational status of epithelioid/mixed GISTs associates with the anatomical site of the tumor.
\end{abstract}

Modern Pathology (2004) 17, 889-894, advance online publication, 21 May 2004; doi:10.1038/modpathol.3800136

Keywords: gastrointestinal stromal tumors; KIT; PDGFRA; mutational analysis; anatomic site

Gastrointestinal stromal tumors (GISTs) are tumors of mesenchymal origin that occur primarily in the gastrointestinal tract. The most common site is the stomach followed by the small intestine, rectum, and colon. ${ }^{1-3}$ Occasionally, primary GISTs have been reported outside the gastrointestinal tract, specifi-

Correspondence: Dr M Debiec-Rychter, MD, PhD, Center for Human Genetics, Catholic University of Leuven, O \& N Gasthuisberg, Herestraat 49, 3000 Leuven, Belgium.

E-mail: Maria.Debiec-Rychter@med.kuleuven.ac.be

Received 11 December 2003; revised 3 March 2004; accepted 4 March 2004; published online 21 May 2004 cally in the omentum, mesenteries, and retroperitoneum. ${ }^{4,5}$ Histologically, these neoplasms are usually composed of spindle cells $(70 \%)$ or less frequently of epithelioid $(20 \%)$ or mixed, epithelioid and spindle cell types $(10 \%) .{ }^{6}$ Lesions of mixed cell type may exhibit a transition between spindle and epithelioid areas, or may have both cell types intermingled throughout the tumor specimen. The biologic basis for the different cell type variants present in GISTs is at present unknown.

The majority of GISTs investigated up to now are CD117-antigen positive by immunohistochemical staining, harboring activating mutations of the KIT 
gene. $^{7-13}$ More recently, mutations in PDGFRA, another member of RTK III family, were reported in subset of wild-type KIT GISTs, ${ }^{14,15}$ indicating alternative oncogenic mechanisms of GISTs induction. Previous studies have suggested that the frequency of KIT mutations in epithelioid type GISTs may be lower than in tumors of spindle cell type. ${ }^{13,16}$ Additionally, all primary GISTs with activating mutations of PDGFRA reported by Hirota et $a l^{15}$ originated from the stomach. It is unclear, however, whether these GISTs carrying PDGFRA mutant isoforms were associated with any specific GIST morphological variant.

In this study, we analyzed the distribution of KIT and PDGFRA mutations in a group of 28 epithelioid and mixed type GIST variants in order to determine the prevalence of KIT/PDGFRA mutations in GISTs with an epithelioid component at different anatomical sites.

\section{Materials and methods}

In total, 28 tumors featuring epithelioid or mixed cell type morphology were retrieved out of 102 primary or recurrent GISTs from the Departments of Pathology, University Hospital in Leuven, from the Department of Pathology, University of Maastricht, and from the Department of Biology and Genetics, Medical University of Gdansk.

Histopathologic examination was performed on tissue fixed in $10 \%$ formalin and embedded in paraffin. Histopathological sections $(5 \mu \mathrm{m})$ were cut from the paraffin blocks for routine hematoxylin/ eosin (H\&E) and immunohistochemical staining (avidin-biotin-peroxidase complex method) using the following monoclonal (mc), and polyclonal (pc) antibodies: CD117 (pc, 1/250, DAKO, Glostrup, Denmark), CD34 (mc, 1/10, Becton Dickinson, San Jose, CA, USA), $\alpha$-SMA (mc, 1/100, DAKO, Glostrup, Denmark), desmin (mc, 1/20, ICN, Aurora, OH, USA), and S-100 (pc, 1/300, DAKO, Glostrup, Denmark). The CD117 staining was performed without antigen retrieval. The presence of mast cells in all cases served as an internal control for CD117 staining. The categorization to histopathologic subtype was based on standard and widely accepted criteria. ${ }^{6}$ Proliferative activity was evaluated by counting mitoses per 50 high-power fields (HPFs). The assessment of the risk of malignant behavior was performed according to Fletcher et $a l^{6}$ into low risk (tumors with a diameter of $2 \mathrm{~cm}$ or less and no more than 5 mitoses per $50 \mathrm{HPFs}$ ), intermediate risk (tumors with a diameter of $5 \mathrm{~cm}$ or less and no more than 10 mitoses per $50 \mathrm{HPFs}$ or tumors with a diameter of $5-10 \mathrm{~cm}$ and less than 5 mitoses per 50 HPFs), and high risk (all other).

\section{Molecular Studies}

Genomic DNA from the tumor tissues was extracted from paraffin-embedded tissue using microdissec- tion procedure, as previously described. ${ }^{12}$ All 102 cases were screened for activating KIT mutations within exons 9, 11, 13, and 17. In addition, all epithelioid and mixed cell type GISTs were screened for PDGFRA mutations within exons 12 and 18. Polymerase chain reaction (PCR) amplification was performed using AmpliTaq Gold DNA polymerase and the GeneAmp ${ }^{\circledR}$ PCR System 2400 (Applied Biosystems, Foster City, CA, USA). PCR mixture and conditions were standard as recommended by Applied Biosystems. Primer sequences were obtained from the genetic databases available online through National Center for Biotechnology Information (NCBI) (http://www.ncbi.nlm.nih.gov). In some cases, according to the quality of DNA, a second round of amplification was performed using nested primers. All of the primer sequences, annealing temperatures, and expected product sizes are shown in Table 1. The PCR products were identified by agarose gel electrophoresis using a $2 \%$ agarose gel. The PCR products were purified with the Microcon PCR purification columns (Millipore, MA, USA) and screened for mutations by denaturing high-pressure liquid chromatography (DHPLC) using the WAVE system (Transgenomic Inc., UK). Samples showing an aberrant elution profile were reamplified and sequenced in both directions on an ABI PRISM 3100 Avant sequencer using the BigDye Terminators v. 1.1 cycle sequencing kit (Applied Biosystems).

\section{Results}

The clinical data, and the morphological and genotypic features of all 28 epithelioid/mixed cell type GISTs under the study are summarized in Table 2.

There were 16 male (57.1\%) and 12 female $(42.9 \%)$ patients included in the study. Patients age ranged from 39 to 79 years (median, 60 years). All GISTs were primary tumors, which originated from the stomach $(n=16 ; 57.1 \%)$, duodenum $(n=6$; $21.4 \%)$, small intestine $(n=2 ; 7.4 \%)$, mesenterium $(n=1 ; 3.7 \%)$, colon $(n=1 ; 3.7 \%)$, prostate $(n=1$; $3.7 \%)$, or peritoneum $(n=1 ; 3.7 \%)$. In all, 17 tumors were overtly clinically malignant, showing either recurrence or metastasis, and 13 were primary, nonmalignant.

\section{Histopathologic Data}

Of the 28 GISTs, 14 (50.0\%) had an epithelioid morphology, being composed of uniformly rounded cells with abundant, clear to eosinophilic cytoplasm, and round or oval nuclei (Figure 1a). The remaining 14 other tumors were classified as mixed variants, consisting of cells with typical features of either spindle-shaped or epithelioid type (Figure $1 \mathrm{~b}$ and $\mathrm{c}$ ).

Most of the tumors $(n=24 ; 85.7 \%)$ were positive for CD117. In four tumors (cases 4, 7, 11, and 12), 
Table 1 PCR primers sequence used, with the corresponding annealing temperatures $\left(\mathrm{T}_{\mathrm{A}}\right)$ and expected PCR size products

\begin{tabular}{|c|c|c|c|c|}
\hline Gene & Primers & Primer sequences $5^{\prime} \rightarrow 3^{\prime}$ & $T_{A}\left({ }^{\circ} \mathrm{C}\right)$ & Product sizes (bp) \\
\hline \multirow[t]{12}{*}{ KIT } & Ex9-F1 & CCA CAT CCC AAG TGT TTT ATG & 56 & 352 \\
\hline & Ex9-F2 & CCC CTC CTA GAG TAA GCC AGG GCT T & & \\
\hline & Ex9-R & GGT GTG ATG CAT GTA TTA CCA G & & \\
\hline & Ex11-F & GAT GAT TCT GAC CTA CAA AT & 56 & 299 \\
\hline & Ex11-R1 & AGG AAG CCA CTG GAG TTC CTT & & \\
\hline & Ex11-R2 & CCC CGT CAC TGT TAT GTG TAC CCA & & \\
\hline & Ex13-F1 & GTA TGG TAC TGC ATG CGC TT & 56 & 294 \\
\hline & Ex13-F2 & GCT TGA CAT CAG TTT GCC AG & & \\
\hline & Ex13-R & GAG AAC AAC AGT CTG GGT AA & & \\
\hline & Ex17-F & TTC ACT CTT TAC AAG TTA AAA TG & 56 & 212 \\
\hline & Ex17-R1 & GAA ACT AAA AAT CCT TTG CAG & & \\
\hline & Ex17-R2 & GGA CTG TCA AGC AGA GAA TG & & \\
\hline \multirow[t]{4}{*}{ PDGFRA } & Ex12-F & AAG CTC TGG TGC ACT GGG ACT T & 65 & 251 \\
\hline & Ex12-R & ATT GTA AAG TTG TGT GCA AGG GA & & \\
\hline & Ex18-F & TAC AGA TGG CTT GAT CCT GAG T & 60 & 212 \\
\hline & Ex18-R & AGT GTG GGA GGA TGA GCC TG & & \\
\hline
\end{tabular}

Table 2 Clinical data, histomorphological and genotypic features of 28 epithelioid/mix type GISTs under the study

\begin{tabular}{|c|c|c|c|c|c|c|c|c|}
\hline \multirow[t]{2}{*}{ No. } & \multirow[t]{2}{*}{ Sex/age (years) } & \multirow[t]{2}{*}{ Location } & \multirow[t]{2}{*}{ Malignancy } & \multirow[t]{2}{*}{ Tyре } & \multicolumn{2}{|c|}{ Immunostaining } & \multicolumn{2}{|c|}{ Genotype } \\
\hline & & & & & $C D 117$ & CD34 & KIT & PDGFRA \\
\hline 1 & $\mathrm{~F} / 48$ & St. & Liver meta. & Epith. & + & + & WT & WT \\
\hline 2 & $\mathrm{~F} / 39$ & St. & Malignant & Epith. & + & + & WT & WT \\
\hline 3 & $\mathrm{M} / 64$ & St. & High risk & Epith. & + & - & WT & WT \\
\hline $4^{a}$ & $\mathrm{M} / 59$ & St. & High risk & Epith. & - & + & WT & WT \\
\hline 5 & $\mathrm{M} / 58$ & St. & High risk & Epith. & + & + & WT & WT \\
\hline 6 & $\mathrm{~F} / 64$ & St. & Intermed. risk & Epith. & + & + & WT & WT \\
\hline $7^{\mathrm{a}}$ & $\mathrm{M} / 72$ & St. & High risk & Epith. & - & - & WT & Del 0DIM842-844 \\
\hline 8 & $\mathrm{M} / 79$ & St. & Intermed. risk & Epith. & + & + & WT & D842V \\
\hline 9 & $\mathrm{M} / 49$ & St. & High risk & Epith. & + & - & WT & $\mathrm{D} 842 \mathrm{~V}$ \\
\hline 10 & $\mathrm{M} / 60$ & St. & Liver meta. & Mixed & + & - & WT & D842V \\
\hline $11^{\mathrm{a}}$ & $\mathrm{M} / 61$ & St. & Malignant & Mixed & - & + & WT & D842V \\
\hline $12^{\mathrm{a}}$ & $\mathrm{F} / 62$ & St. & High risk & Mixed & - & + & WT & D842V \\
\hline 13 & $\mathrm{~F} / 77$ & St. & High risk & Mixed & + & + & WT & Del DIMH842-845 \\
\hline 14 & $\mathrm{~F} / 59$ & St. & Low risk & Mixed & + & + & PM V560D & WT \\
\hline 15 & $\mathrm{~F} / 63$ & St. & Malignant & Mixed & + & - & Del WK557-558 & WT \\
\hline 16 & $\mathrm{~F} / 59$ & St. & Perit. meta. & Mixed & + & + & Del EV554-555 & WT \\
\hline 17 & $\mathrm{M} / 51$ & Colon & High risk & Epith. & + & - & Del KPMYEV550-555F & WT \\
\hline 18 & $\mathrm{M} / 48$ & Intra-abd. & Malignant & Epith. & + & + & Del WK557-558E & WT \\
\hline 19 & $\mathrm{~F} / 58$ & Duodenum & Malignant & Epith. & + & - & Del WK557-558 & WT \\
\hline 20 & $\mathrm{~F} / 72$ & Duodenum & Malignant & Epith. & + & + & Del V559 & WT \\
\hline 21 & $\mathrm{M} / 61$ & Duodenium & High risk & Epith. & + & + & PM N822K & WT \\
\hline 22 & $\mathrm{M} / 70$ & Sm. int. & Malignant & Mixed & + & + & PM V559D & WT \\
\hline 23 & $\mathrm{~F} / 70$ & Sm. int. & Malignant & Mixed & + & - & Ins AY502-503 & WT \\
\hline 24 & $\mathrm{M} / 56$ & Duodenum & Malignant & Mixed & + & + & Ins AY502-503 & WT \\
\hline 25 & $\mathrm{M} / 53$ & Duodenum & Malignant & Mixed & + & - & Del VYIDPTQL569-576 & WT \\
\hline 26 & $\mathrm{M} / 68$ & Prostate & High risk & Mixed & + & - & PM V559D & WT \\
\hline 27 & $\mathrm{M} / 56$ & Intra-abd. & Liver meta. & Mixed & + & + & Del WK557-558 & WT \\
\hline 28 & $\mathrm{~F} / 46$ & Duodenum & Liver meta. & Mixed & + & - & WT & WT \\
\hline
\end{tabular}

Abbreviations: St.=stomach; Sm. int.=small intestine; Intra-abd. =intra-abdominal, Perit. = peritoneal; Intermed. =intermediate; meta $=$ metastasis; Epith. $=$ epithelioid; $\mathrm{WT}=$ wild type.

${ }^{\mathrm{a}}$ Previously described. ${ }^{19}$

CD117-immunoreactivity was uniformly absent although CD117-immunopositivity of mast cells, which served as internal control, was preserved.
The use of antigen retrieval did not influence the staining pattern, except for a more intense staining of the mast cells. CD34 was expressed in $17(60.9 \%)$ 
892
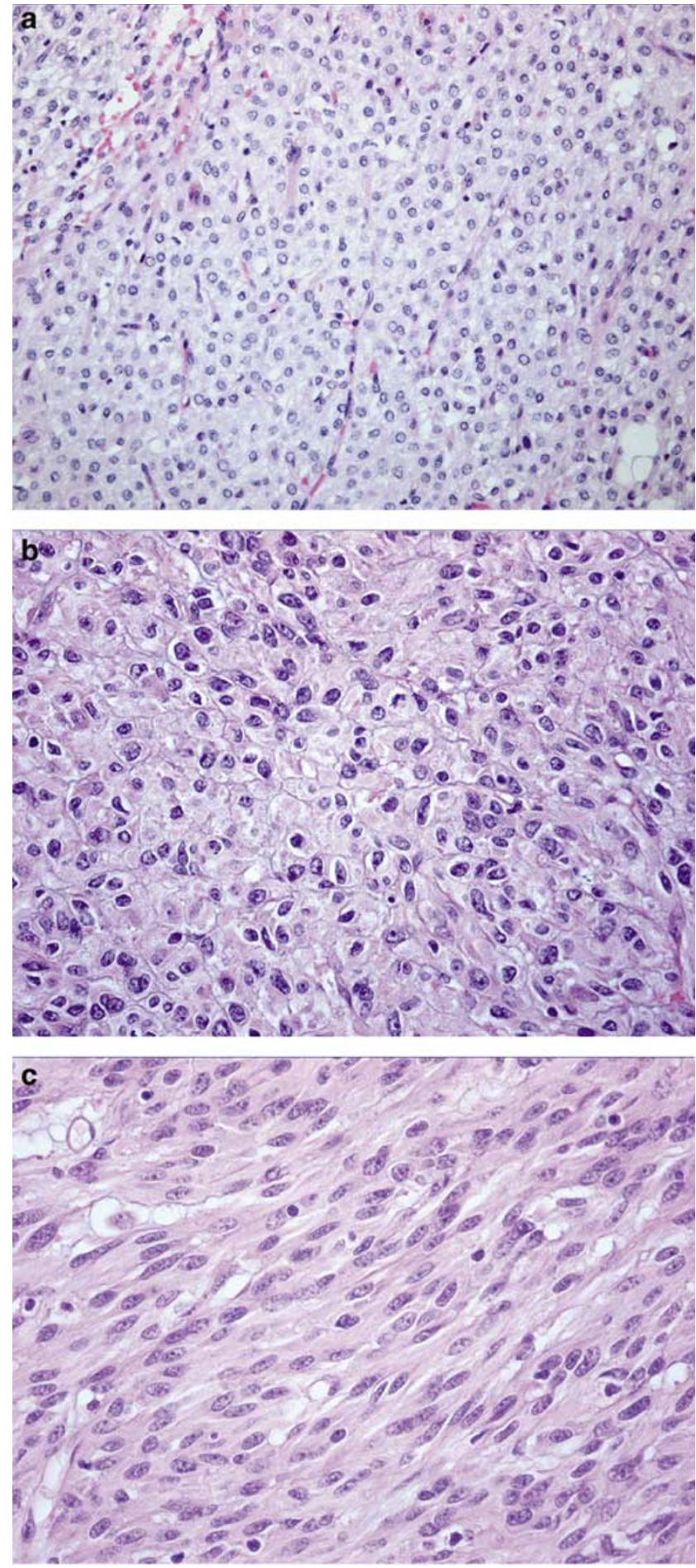

Figure 1 Case 2. Epithelioid GIST. H\&E staining (a); Case 13 Mixed cell type GIST, showing epithelioid (b) and spindle cell areas (c). H\&E staining.

cases. Three tumors (cases 4, 11, and 12) were negative for CD117 but positive for CD34 antigen. One tumor (case 7) was completely negative for both CD117 and CD34 immunoreactivity. None of the tumors showed desmin or S-100 expression.
Based on the size and mitotic activity, 10 out of 13 primary nonmalignant tumors were classified as high-risk tumors; the remaining belonged to low $(n=1)$ - or intermediate $(n=2)$ - risk categories. ${ }^{6}$

\section{Mutational Analysis}

Overall, 80 out of 102 (78.4\%) tumors harbored KIT mutations. The frequency of GISTs harboring KIT mutations differed significantly in spindle cell vs mixed vs epithelioid cell type variants, being $89.1 \%(n=66), 64.3 \%(n=9)$, and $35.7 \%(n=5)$, respectively.

Within epithelioid/mixed cell type group, 11 cases carried exon 11 KIT mutations - either in-frame deletions (six tumors), missense point mutations (three cases) or deletions associated with single base substitution (two cases). Previously described exon 9 AY 502-503 tandem duplication was found in two cases. In addition, one tumor disclosed exon 17 N822 K single amino-acid substitution. The incidence of KIT mutations relied on the tumor anatomic site, being lower in gastric GISTs (three out of $16 ; 18.8 \%$ ) than in tumors of nongastric origin (11 out of $12 ; 91.7 \%$ ) (Table 3 ).

In epithelioid/mixed cell type group, mutations of PDGFRA were observed in seven $(25.0 \%)$ cases; all were in-frame mutations within exon 18. Five tumors disclosed D842V missense mutations, while two other tumors showed either deletion DIMH842845 or DIM842-844, respectively (Figure 2). All GISTs with PDGFRA mutations originated from the stomach.

Seven epithelioid/mixed cell type GISTs did not show detectable KIT or PDGFRA mutations. Majority of these tumors (six out of seven; 85.7\%) originated from the stomach.

\section{Discussion}

Epithelioid and mixed, spindle and epithelioid cell variants of GISTs comprise around $30 \%$ of these neoplasms. ${ }^{6}$ It is also recognized that epithelioid features occur more often in tumors that originate from the stomach than from other location. ${ }^{17}$ So far, the biological factors underlying the existence of morphologic variants of GISTs are unknown. In this study, we evaluated the presence of KIT/PDGFRA mutations in a group of 28 epithelioid and mixed cell type GISTs from the stomach and from nongastric locations, to yield more light on their biology. This group was a part of a larger GISTs cohort (102 cases), with the remaining specimens showing spindle cell GIST features. While overall frequency of GISTs harboring KIT mutations in the whole set of 102 examined cases was $78.4 \%$, the frequency of KIT mutations in tumors with the spindle or mixed type morphology was significantly higher than in the predominantly epithelioid type variant, being 89.1, 64.3, and $35.7 \%$, respectively. The detected 
Table 3 Distribution of KIT/PDGFRA mutations in 28 epithelioid/mixed GISTs according to histologic type and anatomic site of the tumors

\begin{tabular}{|c|c|c|c|c|}
\hline \multirow[t]{2}{*}{ Genotype } & \multicolumn{2}{|c|}{ Histologic type n (\%) } & \multicolumn{2}{|c|}{ Anatomic site $\mathrm{n}(\%)$} \\
\hline & Epithelioid $\mathrm{n}=14$ & Mixed $\mathrm{n}=14$ & Stomach $\mathrm{n}=16$ & Non-stomach $\mathrm{n}=12$ \\
\hline PDGFRA-mutant $n=7$ & $3(21.4)$ & $4(28.6)$ & $7(43.8)$ & 0 \\
\hline Wild type $n=7$ & $6(42.9)$ & $1(7.1)$ & $6(37.5)$ & $1(8.3)$ \\
\hline
\end{tabular}

a

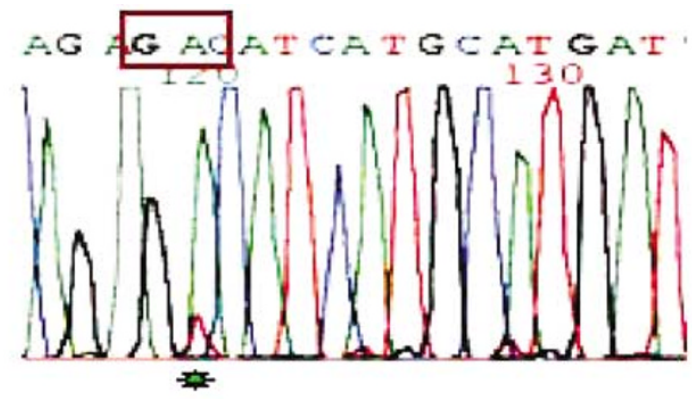

b

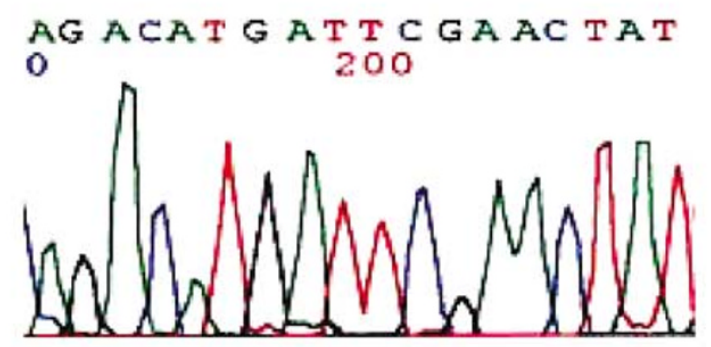

C
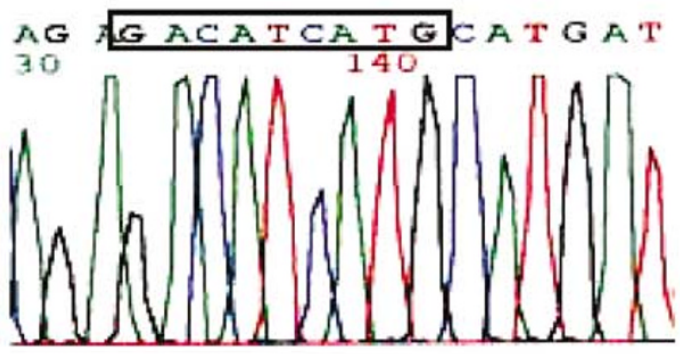

\begin{tabular}{|c|c|c|c|c|c|c|c|}
\hline Codon & 841 & 842 & 843 & $B 44$ & $\theta 45$ & 846 & 847 \\
\hline Sequence & $\hat{A} G A$ & GAC & $A T C$ & ATG & CAT & GAT & $\mathrm{TCG}$ \\
\hline minoacid & $\mathrm{R}$ & $D$ & I & $M$ & H & D & $\mathrm{J}$ \\
\hline
\end{tabular}

Figure 2 The automated sequences data from a GIST heterozygous for PDGFRA exon 18 missense D842 V mutation in case 8 (a). The sequencing data from a GIST showing PDGFRA exon 18 deletion DIMH842-844 in case 7. The amplicons prepared from a GIST heterozygous for the deletion were individually cloned and sequenced to confirm the nature of the mutation (b); PDGFRA exon 18 wild-type clone sequence (c).

mutations within KIT gene were in majority of cases within exon 11, all being either in-frame deletions, point mutations, or deletions associated with amino-acid substitutions. The other KIT mutations included exon 9 AY 502-503 duplication and exon 17 N822 K missense mutation, both being previously described in GISTs. ${ }^{9,10,18}$ Activating mutations within the KIT gene were detected in $57-92 \%$ of GISTs in earlier reports. ${ }^{9-13,16}$ The frequency of KIT mutations in our series was apparently lower in epithelioid than in mixed and spindle type GISTs. Previous reports have already suggested that the frequency of KIT mutations in epithelioid GISTs may be lower than in spindle cell variants. Accordingly, Wardelmann et $a l^{16}$ reported no KIT mutations in seven GISTs with an epithelioid component, while all 21 GISTs with detectable KIT mutations displayed a spindle cell phenotype. This finding was supported by a more recent study ${ }^{13}$ that included 19 epithelioid GISTs and only six of which harbored KIT mutations. Moreover, in the same report only one of nine gastric epithelioid GISTs showed KIT mutation, suggesting that not only histopathology but also tumor site may affect KIT mutation frequency. In support of this notion, only three of $14(21.4 \%)$ GISTs with detectable KIT mutations in our series originated from the stomach, while the others were located elsewhere. Since the frequency of KIT mutations in tumors from nongastric sites in our study was very high $(91.6 \%$; 11 out of 12 tumors), the differences in distribution of KIT mutants within GIST in gastric and nongastric sites cannot be attributed to the potential problems in mutations detection.

Recently, PDGFRA exon 12 and 18 activating mutations were found in a group of GISTs that lack mutations of $K I T,^{14,15}$ bearing similar biological consequences for GISTs biology. However, up-todate studies did not reflect on an association, if any, of PDGFRA mutant GISTs with a specific histopathologic phenotype. Interestingly, however, all primary GISTs with activating mutations of PDGFRA reported by Hirota et $a l^{15}$ originated from the stomach. In our series, activating mutations within the PDGFRA gene were detected in $25 \%$ of our cases; the frequency being substantially higher than $3.9 \%$ frequency reported in the overall GISTs population. ${ }^{14}$ None of the examined tumors harbored mutations of both, KIT and PDGFRA genes, confirming conclusions of Heinrich et $a l^{14}$ that mutations of PDGFRA and KIT are mutually exclusive events in GISTs. All PDGFRA mutations in our series were located within exon 18; five tumors showed Asp $\rightarrow \mathrm{Val}^{842}$ missense point mutations and two others disclosed either four 
(DIMH842-845) or three (DIM842-844) amino-acid deletions. No tumors with PDGFRA exon 12 mutations were found; whether GISTs with an epithelioid phenotype less frequently carry these isoforms has to be confirmed on a larger subset of tumors. Significantly, all seven tumors with detectable PDGFRA mutations in our study originated from the stomach, which yields a $43.7 \%(7 / 16)$ frequency rate of PDGFRA mutants within the group of gastric tumors with epithelioid/mixed type phenotype. Interestingly, three out of seven of these gastric GISTs harboring PDGFRA mutations showed a uniform CD117 immunonegativity, in concordance with their mutational status. The low to undetectable expression of KIT in GISTs harboring PDGFRA mutations was previously described. ${ }^{14}$

In summary, our findings indicate that the frequency of PDGFRA mutations in epithelioid/ mixed GISTs variants is high. In addition, the KIT/ PDGFRA mutational status of these tumors associates with the anatomical site of the tumor. A subset of GISTs with epithelioid features does not harbor KIT/PDGFRA mutations. The genetic events leading to the induction and formation of these tumors are still to be defined.

\section{Acknowledgements}

This paper presents research results of the Belgian program on Interuniversity Poles of Attraction initiated by the Belgian Sate, Prime Minister's Office, Science Policy Programming. Its authors assume the scientific responsibility. We acknowledge the COST support through the COST ACTION B19 'Molecular cytogenetics of solid tumors' in carrying out this work. Bartosz Wasag is supported by Marie Curie European Community fellowship (Contract HPMT-CT2001-00273). Frederik Claessens and Wim DeKelver are acknowledged for their excellent technical assistance.

\section{References}

1 Emory TS, Sobin LH, Lukes L, et al. Prognosis of gastrointestinal smooth muscle (stromal) tumors: dependence on anatomic site. Am J Pathol 1999;23: 82-87.

2 Goldblum JR, Appelman HD. Stromal tumors of the duodenum: A histologic and immunohistochemical study of 20 cases. Am J Surg Pathol 1995;19:71-80.

3 Tworek JA, Goldblum JR, Weiss SW, et al. Stromal tumors of the abdominal colon: a clinicopathologic study of 20 cases. Am J Surg Pathol 1999;23:937-945.

4 Miettinen M, Monihan JM, Sarlomo-Rikala M, et al. Gastrointestinal stromal tumors/smooth muscle
tumors/GISTs in the omentum and mesentry clinicopathologic and immunohistochemical study of 26 cases. Am J Surg Pathol 1999;23:1109-1118.

5 Reith JD, Goldblum JR, Lyles RH, et al. Extragastrointestinal (soft tissue) stromal tumors. An analysis of 48 cases with emphasis on histological predictors of outcome. Mod Pathol 2000;13:577-585.

6 Fletcher CDM, Berman JJ, Corless C, et al. Diagnosis of gastrointestinal stromal tumors: a consensus approach. Hum Pathol 2002;33:459-465.

7 Sarlomo-Rikala M, Kovatich AJ, Miettinen M. CD117: a sensitive marker for gastrointestinal stromal tumors that is more specific than CD34. Mod Pathol 1998; 11:728-734.

8 Hirota S, Isozaki K, Moriyama Y, et al. Gain-of-function mutations of c-kit in human gastrointestinal stromal tumors. Science 1998;279:577-580.

9 Lasota J, Jasinski M, Sarlomo-Rikala M, et al. Mutations in exon 11 of c-kit occur preferentially in malignant versus benign gastrointestinal stromal tumors and do not occur in leiomyomas or leiomyosarcomas. Am J Pathol 1999;154:53-60.

10 Lux ML, Rubin BP, Biase TL, et al. KIT extracellular and kinase domain mutations in gastrointestinal stromal tumors. Am J Pathol 2000;156:791-795.

11 Rubin BP, Singer S, Tsao C, et al. KIT activation is a ubiquitous feature of gastrointestinal stromal tumors. Cancer Res 2001;61:8118-8121.

12 Corless CL, McGreevey L, Haley A. KIT mutations are common in incidental gastrointestinal stromal tumors one centimeter or less in size. Am J Pathol 2002; 160:1567-1572.

13 Antonescu CR, Sommer G, Sarran L, et al. Association of KIT exon 9 mutations with nongastric primary site and aggressive behavior: KIT mutation analysis and clinical correlates of 120 gastrointestinal stromal tumors. Clin Cancer Res 2003;9:3329-3337.

14 Heinrich MC, Corless CL, Duensing A, et al. PDGFRA activating mutations in gastrointestinal stromal tumors. Science 2003;299:708-710.

15 Hirota S, Ohashi A, Nishida T, et al. Gain-of-function mutations of platelet-derived growth factor receptor $\alpha$ gene in gastrointestinal stromal tumors. Gastroenterology 2003;125:660-667.

16 Wardelmann E, Neidt I, Bierhoff E, et al. c-kit mutations in gastrointestinal stromal tumors occur preferentially in spindle rather than in the epithelioid cell variant. Mod Pathol 2002;15:125-136.

17 Trupiano JK, Stewart RE, Misick C, et al. Gastric stromal tumors: a clinicopathologic study of 77 cases with correlation of features with nonaggressive and aggressive clinical behaviors. Am J Surg Pathol 2002; 26:705-714.

18 Lasota J, Wozniak A, Sarlomo-Rikala M, et al. Mutations in exons 9 and 13 of KIT gene are rare events in gastrointestinal stromal tumors: a study of 200 cases. Am J Pathol 2000;157:1091-1095.

19 Debiec-Rychter M, Wasag B, Stul M, et al. Gastrointestinal stromal tumors (GISTs) negative for KIT (CD117 antigen) immunoreactivity. J Pathol 2004;202: 430-438. 